\title{
Estimativas de Tendências e Parâmetros Genéticos do Peso Padronizado aos 378 Dias de Idade, Medidas Corporais e Perímetro Escrotal de Machos Nelore de Sertãozinho, SP1
}

\author{
Joslaine Noely dos Santos Gonçalves Cyrillo², Alexander George Razook ${ }^{3,4}$, \\ Leopoldo Andrade de Figueiredo ${ }^{3}$, Luiz Martins Bonilha Neto ${ }^{3}$, Maria Eugênia Zerlotti Mercadante ${ }^{3}$, \\ Humberto Tonhati ${ }^{5}$
}

RESUMO - Os objetivos deste estudo foram estimar as tendências e os parâmetros genéticos e fenotípicos do peso padronizado aos 378 dias de idade (P378), medidas corporais e perímetro escrotal de machos Nelore, pertencentes às populações selecionadas e controle da Estação Experimental de Zootecnia de Sertãozinho. As herdabilidades, estimadas considerando-se modelo de touro e modelo animal, foram, respectivamente, $0,53 \pm 0,12$ e 0,36 para $\mathrm{P} 378 ; 0,38 \pm 0,11$ e 0,58 para altura na garupa; $0,31 \pm 0,10$ e 0,10 para perímetro torácico; $0,40 \pm 0,11$ e 0,13 para comprimento do corpo; $0,39 \pm 0,11$ e 0,30 para comprimento do dorso; $0,33 \pm 0,10$ e 0,12 para comprimento

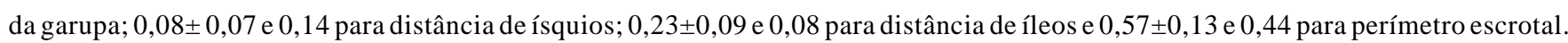
A correlação genética mais alta entre P378 e medidas corporais foi encontrada para perímetro torácico $(0,86 \pm 0,08)$; as demais variaram de 0,46 a 0,72 . Os resultados deste estudo mostraram que, em função dos valores médios a altos das herdabilidades estimadas para P378 e da maioria das características de medidas do corpo dos animais, houve considerável variação genética aditiva nesses atributos. Além disso, devido às altas correlações genéticas de P378 com a maioria das características, pode-se concluir ser bastante provável que grande parte dos genes que controlam o peso pós-desmame também seja responsável pelo desenvolvimento das diferentes regiões do corpo de machos Nelore. A magnitude desses parâmetros justifica a tendência genética positiva nas características de seleção direta e nas secundárias.

Palavras-chave: medidas corporais, parâmetros genéticos, perímetro escrotal, peso pós-desmame, tendência genética

\section{Estimates of Genetic Parameters and Trends for Body Weight at 378 Days, Body Measurements and Scrotal Circumference of Sertãozinho Nellore Males}

\begin{abstract}
The objectives of this study were to estimate the genetic trends and also the genetic and phenotypic parameters for body weight at 378 days (W378), body measurements and scrotal circumferences of Nellore males from selected and control populations of the Estação Experimental de Zootecnia de Sertãozinho (SP), Brazil. The heritability estimates, considering sire and animal model were, respectively, $0.53 \pm 0.12$ and 0.36 for W378 days; $0.38 \pm 0.11$ and 0.58 for hip height; $0.31 \pm 0.10$ and 0.10 for chest girth; $0.40 \pm 0.11$ and 0.13 for body length; $0.39 \pm 0.11$ and 0.30 for dorsal line length; $0.33 \pm 0.10$ and 0.12 for rump length; $0.08 \pm 0.07$ and 0.14 for distance between pin bones; $0.23 \pm 0.09$ and 0.08 for distance between hip bones and $0.57 \pm 0.13$ and 0.44 for scrotal circumference. The highest genetic correlation between W378 and body measurements was found for chest girth ( $0.86 \pm 0.08)$, and the others ranged from 0.46 to 0.72. Based on the median to high heritability values found for the majority of traits, it is expected to exist a good amount of additive genetic variability controlling their variation. In addition, due to the highest correlation between W378 and most of the traits, it is probable that most part of the genes that control post-weaning weight might control the growth of different regions of animal body. The good values for the genetic correlation and heritability explain the positive genetic trends for direct and secondary traits in the selection for W378.
\end{abstract}

Key Words: body measurements, genetic trend, genetic parameters, post-weaning weight, scrotal circumference

\section{Introdução}

Estimativas de componentes de (co)variâncias e herdabilidades são parâmetros populacionais essenciais, tanto para a pesquisa, como para a prática em melhoramento genético animal. Esses parâmetros são inerentes a determinada população e podem mudar com o tempo, devido a mudanças em sua estrutura genética e decisões de manejo (KOOTS et al. 1994).

Estimativas de herdabilidade de peso ao redor dos 12 meses são freqüentes na literatura. Trabalhos de revisão realizados por MRODE (1988), MOHIUDDIN (1993), MERCADANTE (1994) e KOOTS et al. (1994) expõem amplamente o assunto.

\footnotetext{
${ }^{1}$ Parte da dissertação de Mestrado apresentada pela $1^{\mathrm{a}}$ autora a FCAVJ - UNESP - Jaboticabal

2 Assistente Técnico de Direção - EEZ de Sertãozinho. E.mail: cyrillo@izsp.br

3 Pesquisador Científico - IZ - EEZ de Sertãozinho. E.mail: sertaozinho@izsp.br

4 Bolsista do CNPq. E.mail: razook@izsp.br

5 Professor Assistente - Depto. Melh. Gen. Ani. - FCAVJ - UNESP - Jaboticabal. Bolsista do CNPq. E.mail: tonhati@fcav.unesp.br
} 
Os resultados encontrados pelos autores, avaliando estimativas obtidas pelos mais diferentes métodos, raças, práticas de manejo e número de indivíduos na amostra, podem ser considerados de moderados a altos, com valores médios de 0,$48 ; 0,24 ; 0,29 \pm 0,002$; e $0,35 \pm 0,22$ respectivamente. No entanto, raros são os trabalhos que avaliam medidas corporais, com exceção de perímetro escrotal, que tem recebido maior atenção por parte de pesquisadores e criadores. Em revisão, CYRILLO (1998) encontrou grande amplitude de valores de estimativas de herdabilidade de medidas corporais e perímetro escrotal em zebuínos, com variação de 0,32 para comprimento do corpo a 0,57 para distância de íleos (FERNANDES et al., 1996) e de 0,02 (FERNANDES et al., 1996) a $0,47 \pm 0,07$ (BERGMAN et al., 1996) para perímetro escrotal. As correlações genéticas entre essas características e pesos ao redor dos 12 meses, encontradas na literatura, apresentaram-se moderadas e altas.

O objetivo do presente trabalho foi estimar parâmetros genéticos e fenotípicos, assim como as tendências anuais do peso padronizado aos 378 dias de idade (P378), medidas corporais e perímetro escrotal de machos Nelore submetidos à seleção para peso pós-desmame, participantes do projeto de melhoramento genético das raças Zebuínas de Sertãozinho.

\section{Material e Métodos}

As informações utilizadas no presente estudo são provenientes dos rebanhos da raça Nelore pertencentes à Estação Experimental de Zootecnia de Sertãozinho, unidade de pesquisa do Instituto de Zootecnia, localizada ao norte do estado de São Paulo, Brasil. A população bovina da raça Nelore da Estação Experimental atualmente é composta de 791 animais puros de origem, sendo 349 matrizes, 58 reprodutores, incluindo reservas e 384 bezerros, garrotes e novilhas, manejados de acordo com o Projeto de Melhoramento Genético das Raças Zebuínas. A metodologia de execução e os detalhes das populações fundadoras do referido projeto estão documentados em CYRILLO et al. (2000).

\section{Procedimento de obtenção das medidas corporais}

As medidas corporais foram tomadas após a pesagem final da prova de ganho de peso (PGP), utilizando-se tronco de contenção e instrumentos métricos apropriados. As normas de execução das Provas de Ganho de Peso de Sertãozinho estão descritas em RAZOOK et al. (1997).
Foram obtidas as seguintes medidas: altura posterior (ATPF): representada por uma vertical baixada da porção anterior do osso sacro ao solo; perímetro torácico (PTOR): tomada pelo contorno do tórax passando pelo cilhadouro e voltando perpendicularmente à linha do dorso; comprimento do corpo $(\mathrm{COM})$ : obtida lateralmente entre a ponta inferior da espádua e a ponta do ísquio; comprimento dorsolombo (DOR): tomada ao longo da área compreendida entre o final da cernelha e início do sacro; comprimento da garupa (GAR): tomada lateralmente entre a ponta do íleo e a ponta do ísquio; distâncias dos ísquios (ISQ): obtida por trás entre as tuberosidades isquiáticas; distância de íleos (ILEO): tomada entre os dois ângulos anteriores externos dos íleos; perímetro escrotal (PE): tomada horizontalmente na porção mediana, equivalente ao perímetro máximo da bolsa escrotal.

Para a maioria das medidas de comprimento, foi utilizada fita métrica padrão. As medidas do PTOR e COM foram tomadas utilizando-se uma corda auxiliar, que era levada à fita métrica após efetuadas as medidas.

As medidas de ISQ e ILEO foram tomadas com paquímetros de tamanhos apropriados.

A ATPF foi obtida por ocasião da pesagem ao final da PGP por meio de observações em fitas métricas instaladas no interior das balanças. O perímetro escrotal foi tomado com fita métrica metálica apropriada.

\section{A amostra populacional utilizada}

Para formação do arquivo de dados base, foram considerados registros relativos a 908 bovinos machos da raça Nelore, pertencentes aos rebanhos Nelore Controle $(\mathrm{NeC})$, Nelore Seleção $(\mathrm{NeS})$ e Nelore Tradicional (NeT) da Estação Experimental de Zootecnia de Sertãozinho, nascidos entre os anos de 1989 e 1996, filhos de 83 touros e 466 matrizes.

Para a análise conduzida, empregando um modelo animal, foi utilizado um arquivo de pedigree, contendo 4459 informações, registradas no período de 1958 a 1996, das quais 385 são de animais base e o restante, de indivíduos com genealogia completa.

\section{Análises estatísticas}

As análises para obtenção de estimativas de componentes de (co)variâncias e constantes de ajustes foram conduzidas, primeiramente, utilizando-se modelo de touro, baseado no método dos quadrados mínimos, para dados com frequências desiguais nas subclasses, em um modelo hierárquico com touro 
aninhado dentro de rebanhos. Os componentes de (co)variância associados aos efeitos genéticos foram estimados simultaneamente às avaliações dos efeitos fixos, utilizando-se o modelo MTY=3 (HARVEY, 1990).

Após efetuadas análises preliminares, foram definidos modelos específicos para cada uma das características estudadas.

Foram utilizados somente registros de touros com número de filhos superior a quatro. $\mathrm{O}$ modelo 1 , utilizado para obtenção dos parâmetros genéticos das características P378 e ATPF, foi o seguinte:

$$
y_{i j k m}=\mu+a_{i}+r_{j}+t r_{j k}+m_{l}+b_{1}(X-\bar{X})+b_{2}(X-\bar{X})^{2}+e_{i k l m}
$$

em que $\mathrm{y}_{\mathrm{ijklm}}=$ valores observados para as características P378 e ATPF; $\mu=$ constante comum a todos os indivíduos; $\mathrm{a}_{\mathrm{i}}=$ efeito fixo de ano de realização da PGP ( $i=90$ - 97); $r_{j}=$ efeito fixo de rebanho $(j=\mathrm{NeC}$, $\mathrm{NeS}$ e NeT); $\operatorname{tr}_{\mathrm{jk}}=$ efeito aleatório de touro dentro de rebanho; $\mathrm{m}_{1}=$ efeito fixo da idade da mãe em anos $(1=3-10) ; b_{1}$ e $b_{2}=$ coeficientes de regressão linear e quadrático; $\bar{X}=$ valor médio da idade do bezerro ao final da PGP; $\mathrm{e}_{\mathrm{ijklm}}=$ erro aleatório associado a cada observação, e $\sim \mathrm{N}\left(0, \sigma^{2}\right)$.

O modelo 2, utilizado para análise de PTOR, COM, DOR, GAR, ISQ, ILEO e PE, foi similar ao 1, excluindo a idade da mãe, que não apresentou significância em nível de 5\%.

Os coeficientes de herdabilidade, correlações genéticas e fenotípicas e seus respectivos errospadrão foram estimados usando o procedimento descrito por HARVEY (1990), utilizando como relação média entre indivíduos de uma família o valor 0,25 , ou seja, meios - irmãos paternos.

Por este procedimento, se houver efeitos de dominância, epistasia e ambiente permanente, a variância devida a estas fontes de variação será incluída em $\hat{\sigma}_{\mathrm{e}}^{2}$.

Foram também avaliadas as tendências fenotípicas anuais de todas as características, para cada rebanho, utilizando-se o modelo $\mathrm{MTY}=1$ (HARVEY,1990) e considerando os efeitos fixos de ano dentro de rebanho (criado pela opção COMBI$\mathrm{NE}$ ), regressão linear e quadrática sobre a idade do bezerro ao final da PGP e idade da mãe para as características P378 e ATPF.

O modelo 3, utilizado para obtenção das tendências fenotípicas, foi:

$$
\mathrm{y}_{\mathrm{ijkl}}=\mu+a_{j i}+m_{k}+b_{1}(X-\bar{X})+b_{2}(X-\bar{X})^{2}+e_{i j k l}
$$

em que $\mathrm{y}_{\mathrm{ijkl}}=$ valores observados para as caracterís- ticas ATPF e P378; $\mathrm{m}_{\mathrm{k}}$ = efeito fixo de idade da mãe; $\mathrm{a}_{\mathrm{ji}}=$ Efeito fixo de ano de realização da PGP $(i=90 \ldots . .97)$ dentro de rebanho $(j=\mathrm{NeC}, \mathrm{NeS}$ e NeT $)$.

Para as demais características, foi utilizado o mesmo modelo 3, excluindo-se o efeito de idade da mãe.

Estimativas de herdabilidade e correlações genéticas foram obtidas também por meio de análises bivariadas utilizando o programa MTDFREML (BOLDMAN et al., 1993). Os valores de (co)variâncias iniciais foram tomados das análises realizadas inicialmente pelo programa LSMLMW (HARVEY 1990).

Os efeitos de ano e rebanho foram combinados para obter uma identificação única para grupo contemporâneo, o qual foi considerado como único efeito fixo, exceto para P378 e ATPF, que tiveram idade da mãe como segundo efeito fixo. A covariável idade ao final da PGP foi incluída na análise de todas as características.

O modelo animal utilizado, pode ser representado por:

$$
y_{i j k m}=\mu+G_{i}+c_{j}+m_{K}+b_{1}(X-\bar{X})+b_{2}(X-\bar{X})^{2}+e_{i j k m}
$$

em que $\mathrm{y}_{\mathrm{ijklm}}=$ valores observados para as características P378 e ATPF; $\mu=$ constante comum a todos os indivíduos; $\mathrm{G}_{\mathrm{i}}=$ valor genético do animal $\mathrm{i}$; $c_{j}=$ efeito fixo de grupo contemporâneo $(j=$ ano de realização da PGP e rebanho); $\mathrm{m}_{\mathrm{k}}=$ efeito fixo de idade da mãe em anos $(\mathrm{k}=3-10) ; \mathrm{b}_{1}$ e $\mathrm{b}_{2}=$ coeficientes de regressão linear e quadrático; $\mathrm{X}=$ idade do bezerro ao final da PGP; $\bar{X}=$ valor médio da idade do bezerro ao final da PGP; $\mathrm{e}_{\mathrm{ijklm}}=$ erro aleatório associado a cada observação, e $\sim \mathrm{N}\left(0, \sigma^{2}\right)$.

\section{Tendências genéticas anuais}

As tendências genéticas anuais foram estimadas considerando-se dois métodos:

a) A diferença entre os coeficientes de regressão linear das médias anuais de cada caráter, em função de anos, para cada rebanho selecionado, obtido pelo modelo 3, em relação ao rebanho controle.

b) Desvios absolutos dos rebanhos selecionados, em relação ao rebanho controle, considerando-se os valores médios, para os oito anos de progênies, e os coeficientes e intervalos de geração médios de acordo com: $\triangle G /$ ano $=\frac{D E S V}{\overline{I G} x \overline{C G}}$

em que DESV = diferença das médias entre o rebanho seleção e o rebanho controle; $\overline{I G}=$ intervalo de geração médio das progênies de 89 a 96; $\overline{C G}=$ coeficiente de geração médio dessas progênies. 


\section{Resultados e Discussão}

\section{Correlações genéticas}

$\mathrm{Na}$ Tabela 1 estão apresentadas as correlações genéticas e fenotípicas entre as características estudadas.

A maioria das estimativas de correlações genéticas entre P378 e características de dimensões corporais foi alta e a totalidade, positiva, variando de 0,46 a 0,86 .

A magnitude das correlações entre P378 e as demais medidas indica que os genes que influenciam P378 têm, também, grande efeito sobre características de medidas corporais. A maior correlação de P378 com as demais características foi observada com PTOR $(0,86)$, seguida de COM $(0,72)$, ILEO e PE $(0,71)$, GAR $(0,64)$, ATPF $(0,61)$ e DOR $(0,46)$. A correlação genética entre P378 e ISQ apresentou valor acima da unidade com alto erro associado, sendo de difícil explicação.

Embora alguns autores afirmem ser a seleção para peso padronizado a determinadas idades a responsável pelo efeito indesejável de excesso de altura em animais zebuínos, pode-se verificar, no presente estudo, que, além da ATPF, outras características de

grande interesse no desenvolvimento corporal, como por exemplo, PTOR e ILEO, são também geneticamente associadas à característica P378. Conforme relatado por CYRILLO et al. (2000), essas características tiveram aumento significativo em relação à população controle como medida da mudança genética correlacionada.

Altas correlações genéticas entre pesos e medidas corporais foram também verificadas por FERNANDES et al. (1996), os quais estudaram pesos e medidas de animais da raça Brahmam, com idades entre 8 e 24 meses, sendo que, valores acima de 0,70 foram encontrados entre pesos e ATPF, PTOR, COM, ISQ e ILEO. O mesmo foi verificado por BROWN et al. (1973), que encontraram valores de 0,82 e 0,75 para correlação genética entre peso ao final do teste de desempenho e ATPF e PTOR, respectivamente.

A alta correlação de PE com P378 $(0,71)$ encontrada no presente trabalho é inferior ao valor de $0,91 \pm 0,07$ encontrado por ALENCAR et al. (1993), em estudo de um rebanho da raça Canchim. Essa alta correlação observada entre pesos e PE sugere que touros selecionados para peso, provavelmente, terão filhos com testículos de maiores perímetros e, consequientemente, com características reprodutivas desejáveis.

Tabela 1 - Herdabilidades na diagonal, correlações genéticas $\left(r_{g}\right)$ acima da diagonal e fenotípicas $\left(r_{p}\right)$ abaixo da diagonal, entre peso padronizado aos 378 dias, medidas corporais e perímetro escrotal de machos Nelore ao final da PGP

Table 1 - Heritability on diagonal, genetic correlation $\left(r_{g}\right)$ above the diagonal, and phenotypic correlation below the diagonal, among body weight, body measurements and scrotal circumference of Nellore males at the end of PGP

\begin{tabular}{|c|c|c|c|c|c|c|c|c|c|}
\hline $\begin{array}{l}\text { Característica }^{1} \\
\text { Traits }^{1}\end{array}$ & $\begin{array}{l}\text { P378 } \\
\text { W378 }\end{array}$ & $\begin{array}{c}\text { ATPF } \\
H H\end{array}$ & $\begin{array}{l}\text { PTOR } \\
C G\end{array}$ & $\begin{array}{c}\mathrm{COM} \\
B L\end{array}$ & $\begin{array}{c}\mathrm{DOR} \\
D L\end{array}$ & $\begin{array}{c}\text { GAR } \\
R L\end{array}$ & $\begin{array}{l}\text { ISQ } \\
D P B\end{array}$ & $\begin{array}{l}\text { ILEO } \\
D H B\end{array}$ & $\begin{array}{l}\mathrm{PE} \\
S P\end{array}$ \\
\hline P378(W378) & $\begin{array}{c}\mathbf{0 , 5 3} \pm \mathbf{0 , 1 2} \\
\mathbf{0 , 3 6}\end{array}$ & $0,61 \pm 0,12$ & $0,86 \pm 0,08$ & $0,72 \pm 0,12$ & $0,46 \pm 0,16$ & $0,64 \pm 0,14$ & $1,27 \pm 0,45$ & $0,71 \pm 0,14$ & $0,71 \pm 0,13$ \\
\hline $\operatorname{ATPF}(H H)$ & 0,65 & $\begin{array}{c}\mathbf{0 , 3 8} \pm \mathbf{0 , 1 2} \\
\mathbf{0 , 5 8} *\end{array}$ & $0,79 \pm 0,12$ & $0,73 \pm 0,13$ & $0,44 \pm 0,18$ & $0,74 \pm 0,14$ & $0,60 \pm 0,35$ & $0,53 \pm 0,21$ & $0,47 \pm 0,20$ \\
\hline $\operatorname{PTOR}(C G)$ & 0,70 & 0,55 & $\begin{array}{c}\mathbf{0 , 3 1} \pm \mathbf{3 0 , 1 0} \\
\mathbf{0 , 1 0} *\end{array}$ & $0,79 \pm 0,14$ & $0,51 \pm 0,19$ & $0,52 \pm 0,19$ & $0,88 \pm 0,37$ & $0,71 \pm 0,21$ & $0,53 \pm 0,24$ \\
\hline $\operatorname{COM}(B L)$ & 0,48 & 0,42 & 0,40 & $\begin{array}{c}\mathbf{0 , 4 0} \pm \mathbf{0 , 1 1} \\
\mathbf{0 , 1 3} *\end{array}$ & $0,72 \pm 0,15$ & $0,46 \pm 0,19$ & $0,89 \pm 0,40$ & $0,55 \pm 0,22$ & $0,56 \pm 0,19$ \\
\hline $\operatorname{DOR}(D L)$ & 0,33 & 0,36 & 0,27 & 0,24 & $\begin{array}{c}\mathbf{0 , 3 9} \pm \mathbf{0 , 1 1} \\
\mathbf{0 , 3 0} *\end{array}$ & $0,40 \pm 0,20$ & $0,33 \pm 0,37$ & $0,04 \pm 0,32$ & $0,26 \pm 0,24$ \\
\hline $\operatorname{GAR}(R L)$ & 0,47 & 0,43 & 0,40 & 0,33 & 0,21 & $\begin{array}{c}\mathbf{0 , 3 3} \pm \mathbf{3 0 , 1 0} \\
\mathbf{0 , 1 2} *\end{array}$ & $0,73 \pm 0,39$ & $0,12 \pm 0,31$ & $0,45 \pm 0,24$ \\
\hline ISQ $(D P B)$ & 0,48 & 0,35 & 0,38 & 0,30 & 0,25 & 0,29 & $\begin{array}{c}\mathbf{0 , 0 8} \pm \mathbf{0 , 0 7} \\
\mathbf{0 , 1 4} *\end{array}$ & $0,65 \pm 0,41$ & $0,37 \pm 0,36$ \\
\hline $\operatorname{ILEO}(D H B)$ & 0,56 & 0,44 & 0,50 & 0,33 & 0,23 & 0,39 & 0,58 & $\begin{array}{c}\mathbf{0 , 2 3} \pm \mathbf{0 , 0 9} \\
\mathbf{0 , 0 8} *\end{array}$ & $0,65 \pm 0,20$ \\
\hline $\mathrm{PE}(\mathrm{S} P)$ & 0,37 & 0,20 & 0,23 & 0,20 & 0,08 & 0,13 & 0,14 & 0,19 & $\begin{array}{c}0,57 \pm 0,13 \\
0,44^{*}\end{array}$ \\
\hline
\end{tabular}

1 P378: peso padronizado aos 378 dias de idade (kg); ATPF: altura na garupa (cm); PTOR: perímetro torácico (cm); COM: comprimento do corpo (cm); DOR: comprimento dorso-lombo (cm); GAR: comprimento da garupa (cm); ISQ: distância de ísquios (cm); ILEO: distância de íleos (cm); PE: perímetro escrotal $(\mathrm{cm}) .{ }^{*}$ Valores de herdabilidade obtidas por meio de modelo animal.

1 W378: weight standardized at 378 days old (kg); HH: hip height (cm); CG: chest girth (cm); BL: body length (cm); DL: dorsal length (cm); RL: rump length (cm); DPB: distance of pin bones (cm); DHB: distance of hip bones (cm); SP: scrotal circumference (cm). * Heritability values obtained by animal model. 
As correlações genéticas entre as medidas corporais foram, na maioria, moderadas e altas, variando de 0,26 a 0,89 , com valores inferiores para DOR e ILEO $(0,04)$ e GAR e ILEO $(0,12)$. FERNANDES et al. (1996), com animais da raça Brahmam, encontraram valores maiores para correlações entre ATPF, PTOR, COM, ISQ e ILEO, os quais variaram de 0,64 a 0,90.

Os valores encontrados neste estudo sugerem que a seleção para peso (P378), ou para outras características relacionadas ao crescimento, por meio das respostas correlacionadas, resultará em aumentos em outras dimensões corporais.

\section{Correlações fenotípicas}

Todas as correlações fenotípicas entre P378 e as características de medidas corporais foram inferiores às correlações genéticas e de magnitudes medianas (Tabela 1).

As correlações de P378 com ATPF e PTOR foram as que apresentaram maiores magnitudes, sendo os valores encontrados de 0,65 e 0,70 respectivamente.

As correlações fenotípicas entre as medidas corporais apresentaram-se, na maioria, moderadas $(0,13$ a 0,50$)$, com exceção de DOR e PE, que apresentaram baixa correlação $(0,08)$, além de ISQ e ILEO $(0,58)$ e ATPF e PTOR $(0,55)$, para os quais foram obtidos maiores valores.

Correlações fenotípicas altas e positivas entre características não necessariamente significam que a seleção para uma característica levará ao melhoramento da outra, devido ao fato de a correlação fenotípica não ser sempre uma estimativa confiável da relação genética existente entre as características (MOHIUDDIN, 1993).

\section{Herdabilidade}

Estimativas de herdabilidade obtidas por modelo de touro e modelo animal bivariado, para peso, medidas corporais e perímetro escrotal, são apresentadas na Tabela 1.

Todas as características estudadas apresentaram valores baixos ou medianos, considerando-se o modelo de touros.

A estimativa de herdabilidade obtida para P378 $(0,53 \pm 0,12)$ situa-se acima dos valores encontrados na literatura, inclusive acima da média $(0,29 \pm 0,002)$ obtida por Mercadante (1994), em revisão feita somente com trabalhos realizados com zebuínos.

Em estudo anterior, considerando o mesmo plantel, e amostra populacional maior, GONÇALVES et al. (1996) encontraram estimativa de 0,58 $\pm 0,09$, obtida por modelo de touro, valor este próximo ao encontrado no presente estudo, considerando a mesma metodologia de análise.

Para ATPF, no presente trabalho, foi encontrada estimativa de $0,38 \pm 0,11$. Estimativas obtidas para a mesma característica, em estudos realizados com animais de origem européia, variaram de 0,43 $\pm 0,13$ a $0,57 \pm 0,16$. No único trabalho semelhante realizado com zebuínos, MAGNABOSCO et al. (1996) encontraram valor de 0,54 .

Com relação à característica perímetro torácico, o valor encontrado $(0,31 \pm 0,1)$ foi intermediário em relação aos encontrados na literatura, que variaram de 0,11 a $0,40 \pm 0,13$.

Para a característica comprimento do corpo, o valor $0,40 \pm 0,11$ foi superior aos encontrados na literatura, que variaram de 0,25 a 0,38 , para animais de origem européia.

Para a característica DOR foi encontrado valor de $0,39 \pm 0,1$, o qual se encontra abaixo do único valor $(0,46 \pm 0,19)$ encontrado na literatura, em trabalho desenvolvido por LIMA et al. (1989), considerando avaliação subjetiva.

A característica comprimento da garupa apresentou valor de herdabilidade de $0,33 \pm 0,1$, contrastando com o valor $0,49 \pm 0,19$, em trabalho desenvolvido por LIMA et al. (1989).

Para ISQ, o valor encontrado foi $0,08 \pm 0,0$, contrastando com o valor de 0,32 obtido por FERNANDES et al. (1996), em estudo de animais da raça Brahmam. A característica ILEO apresentou valor de $0,23 \pm 0,0$, menor que $0,34 \pm 0,19$ e 0,57 , obtidos por LIMA et al. (1989) e FERNANDES et al. (1996), respectivamente.

A característica PE apresentou valor igual a $0,57 \pm 0,1$, o qual se encontra intermediário entre a grande amplitude de valores $(0,02$ a $0,84 \pm 0,20)$, encontrada na literatura consultada.

No geral, os valores obtidos para as medidas corporais apresentaram-se medianos, exceto para P378 e PE, que apresentaram valores altos e ISQ, que apresentou valor bem abaixo das demais características.

As estimativas obtidas por meio de análise considerando-se modelo animal bivariado, conforme apresentadas na Tabela 1, apresentaram-se, na maioria, inferiores às obtidas considerando-se o modelo de touros, com exceção de altura na garupa e ísquio.

Os valores das estimativas de herdabilidade obtidos no presente estudo, com exceção dos obtidos para ISQ, indicam que a contribuição da variância genética aditiva é suficientemente grande para que se possa 
CYRILLO et al.

proceder com segurança à seleção baseada no fenótipo dos indivíduos para medidas corporais. Entretanto, conforme salientado por WINKLER et al. (1993), deve-se sempre ter em mente que a herdabilidade é um parâmetro referente à determinada população em determinada época, sendo as diferenças entre as estimativas atribuíveis ao tamanho da amostra, à composição genética do rebanho e ao manejo a que é submetida a população.

\section{Tendências fenotípicas e genéticas}

As médias anuais de peso, medidas corporais e perímetro escrotal, em função de ano de realização da PGP, para cada rebanho, estão representadas graficamente na Figura 1. Os gráficos expressam o comportamento fenotípico dessas características em cada rebanho ao longo dos anos.

As equações que explicam esse comportamento para as características, dentro de rebanhos, são apresentadas na Tabela 2, assim como as tendências genéticas anuais obtidas como desvios dos coeficientes de regressão linear entre os rebanhos selecionados e controle.

Consta da Tabela 3 as tendências genéticas anuais para peso, medidas corporais e perímetro escrotal, obtidas, em função dos desvios em relação a $\mathrm{NeC}$ e levando-se em conta o intervalo e coeficiente de geração médio dos oito anos de progênies estudadas de 5,11 anos e 2,9 gerações para o rebanho NeS e

Tabela 2 - Equações de regressão de P378, medidas corporais e perímetro escrotal, em função de ano da PGP e ganho genético por ano (DG/ano)

Table 2 - Regression equation for P378, body measurements and scrotal circumference on year of performance test (PGP) and annual genetic trends (DG/year)

\begin{tabular}{|c|c|c|c|c|c|}
\hline $\begin{array}{l}\text { Característica }^{1} \\
\text { Trait }^{1}\end{array}$ & $\begin{array}{c}\text { Rebanho }^{2} \\
\text { Herd }^{2} \\
\end{array}$ & $\mathrm{~b}_{\mathrm{o}}$ & $\mathrm{b}_{1}$ & $\mathrm{~b}_{2}$ & $\begin{array}{l}\Delta \mathrm{G} / \text { ano } \\
\Delta G / \text { Year }\end{array}$ \\
\hline & $\mathrm{NeC}$ & 305,0 & $0,18^{\mathrm{NS}}$ & $0,028^{\mathrm{NS}}$ & \\
\hline P378 & $\mathrm{NeS}$ & 305,0 & $5,1 * *$ & $0,004^{\mathrm{NS}}$ & 4,92 \\
\hline \multirow[t]{2}{*}{ W378 } & $\mathrm{NeT}$ & 305,0 & $3,2 * *$ & $0,20 * *$ & 3,08 \\
\hline & $\mathrm{NeC}$ & 132,0 & $-0,27 *$ & $0,008^{\mathrm{NS}}$ & \\
\hline ATPF & $\mathrm{NeS}$ & 132,0 & $0,20 *$ & $0,007^{\mathrm{NS}}$ & 0,47 \\
\hline \multirow[t]{2}{*}{$\underline{H H}$} & $\mathrm{NeT}$ & 132,0 & $0,25 * *$ & $-0,012 * *$ & 0,52 \\
\hline & $\mathrm{NeC}$ & 161,0 & $-0,48^{*}$ & $0,015^{\mathrm{NS}}$ & \\
\hline PTOR & $\mathrm{NeS}$ & 161,0 & $0,41 *$ & $0,024^{*}$ & 0,89 \\
\hline \multirow[t]{2}{*}{$C G$} & $\mathrm{NeT}$ & 161,0 & $0,11^{\mathrm{NS}}$ & $-0,03 *$ & 0,59 \\
\hline & $\mathrm{NeC}$ & 122,5 & $-1,66 * *$ & $0,02 *$ & \\
\hline $\mathrm{COM}$ & $\mathrm{NeS}$ & 122,5 & $-0,88 * *$ & $0,02 *$ & 0,78 \\
\hline \multirow[t]{2}{*}{$B L$} & $\mathrm{NeT}$ & 122,5 & $-0,93 * *$ & $-0,02 * *$ & 0,73 \\
\hline & $\mathrm{NeC}$ & 62,6 & $-0,83^{* *}$ & $-0,009^{\mathrm{NS}}$ & \\
\hline DOR & $\mathrm{NeS}$ & 62,6 & $-0,76 * *$ & $-0,026 * *$ & 0,07 \\
\hline \multirow[t]{2}{*}{$D L$} & $\mathrm{NeT}$ & 62,6 & $-0,14^{\mathrm{NS}}$ & $-0,033 * *$ & 0,83 \\
\hline & $\mathrm{NeC}$ & 36,6 & $-0,86 * *$ & $0,004^{\mathrm{NS}}$ & \\
\hline GAR & $\mathrm{NeS}$ & 36,6 & $-0,54 * *$ & $0,01 * *$ & 0,32 \\
\hline \multirow[t]{2}{*}{$R L$} & $\mathrm{NeT}$ & 36,6 & $-0,61 * *$ & $-0,01 * *$ & 0,19 \\
\hline & $\mathrm{NeC}$ & 25,1 & $0,51 * *$ & $0,03 * *$ & \\
\hline ISQ & $\mathrm{NeS}$ & 25,1 & $0,63 * *$ & $0,04 * *$ & 0,12 \\
\hline \multirow[t]{2}{*}{$D P B$} & $\mathrm{NeT}$ & 25,1 & $0,62 * *$ & $0,03 * *$ & 0,11 \\
\hline & $\mathrm{NeC}$ & 36,3 & $-0,05^{\mathrm{NS}}$ & $-0,006^{* *}$ & \\
\hline ILEO & $\mathrm{NeS}$ & 36,3 & $0,19 * *$ & $0,02 * *$ & 0,24 \\
\hline \multirow[t]{2}{*}{$\underline{D H B}$} & $\mathrm{NeT}$ & 36,3 & $0,07^{\mathrm{NS}}$ & $0,01 * *$ & 0,12 \\
\hline & $\mathrm{NeC}$ & 21,9 & $-0,29 * *$ & $-0,007^{\mathrm{NS}}$ & \\
\hline $\mathrm{PE}$ & $\mathrm{NeS}$ & 21,9 & $0,02^{\mathrm{NS}}$ & $-0,0015 * *$ & 0,31 \\
\hline$S P$ & $\mathrm{NeT}$ & 21,9 & $-0,5 * *$ & $-0,02 * *$ & $-0,21$ \\
\hline
\end{tabular}

* $(P<0,05) ;{ }^{* *}(P<0,01)$; NS: não-significativo; $b_{0}$ : intercepto; $b_{1}$ e $b_{2}$ : coeficientes de regressão linear e quadrático.

1 P378: peso padronizado aos 378 dias de idade (kg); ATPF: altura na garupa (cm); PTOR: perímetro torácico (cm); COM: comprimento do corpo (cm); DOR: comprimento dorso-lombo $(\mathrm{cm})$; GAR: comprimento da garupa (cm); ISQ: distância de ísquios (cm); ILEO: distância de íleos (cm); PE: perímetro escrotal (cm).

2 NeC: Rebanho Nelore Controle, NeS: Rebanho Nelore Seleção, NeT: Rebanho Nelore Tradicional.

* $(P<.05) ; *{ }^{*}(P<.01) ; N S$ : Nom significant; $b_{1}$ e $b_{2}$ : linear and quadratics regression coefficients.

1 W378: Weightstandardized at 378 days old (kg); HH: hip height (cm);CG: chestgirth (cm); BL:body length (cm);DL: dorsallength (cm); RL: rump length (cm), DPB: distance of pin bones (cm); DHB: distance of hip bones (cm); SP: scrotal circumference (cm).

2 NeC: Control Herd, NeS: Selection Herd, NeT: Traditional Herd. 
P378

WP378

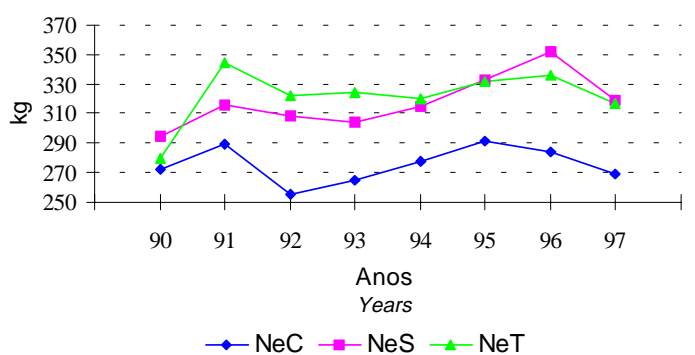

PTOR

$C G$

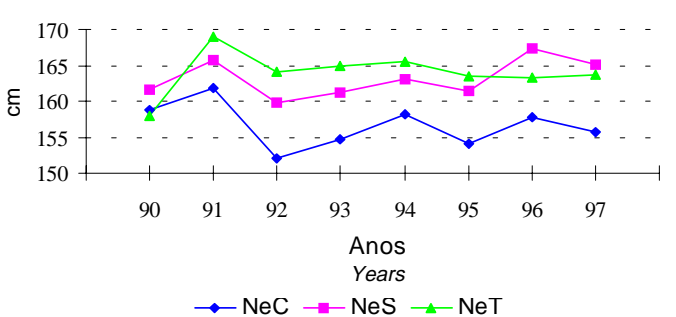

DOR

$D L$

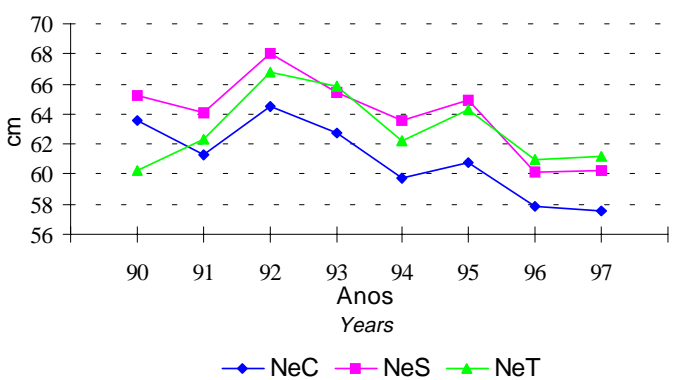

ISQ

$D P B$

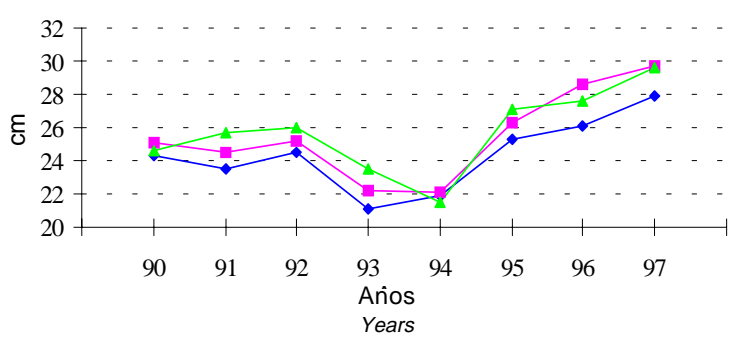

$\rightarrow \mathrm{NeC} \rightarrow \mathrm{NeS} \triangle \mathrm{NeT}$

PE

$E P$

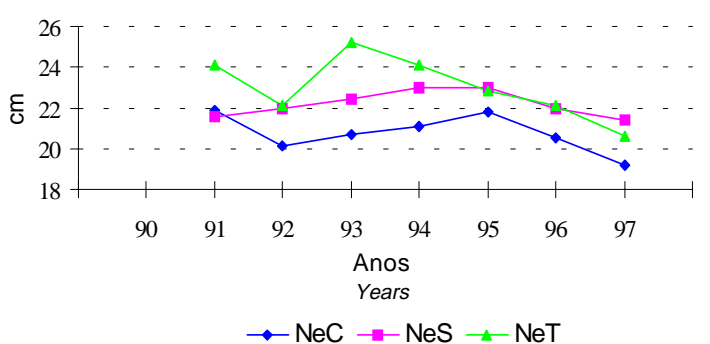

ATPF

$\mathrm{HH}$

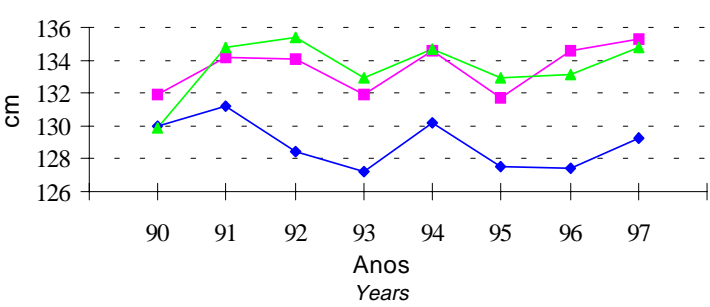

$-\mathrm{NeC} \leadsto-\mathrm{NeS} \leadsto-\mathrm{NeT}$

(O)

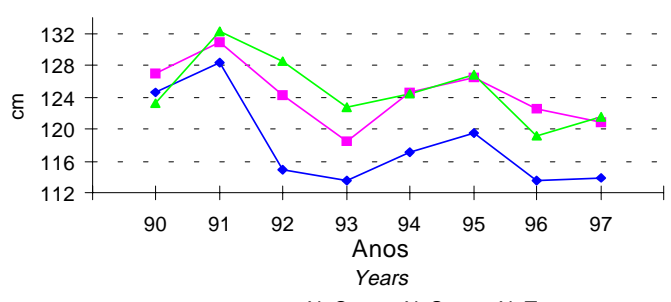

$\rightarrow \mathrm{NeC} \rightarrow-\mathrm{NeS} \leadsto-\mathrm{NeT}$

GAR

$R L$

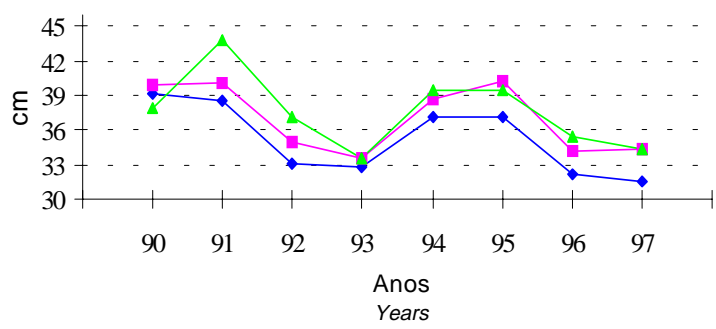

$\rightarrow \mathrm{NeC} \rightarrow-\mathrm{NeS} \leadsto \mathrm{NeT}$

ILEO

$D H B$

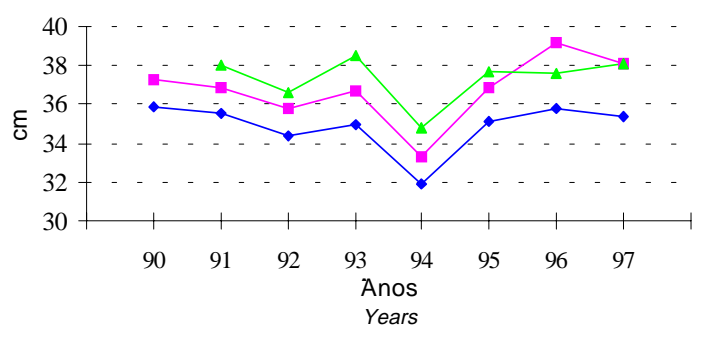

$\rightarrow \mathrm{NeC} \rightarrow \mathrm{NeS} \rightarrow \mathrm{NeT}$

P378: peso padronizado aos 378 dias de idade $(\mathrm{kg})$; ATPF: altura na garupa $(\mathrm{cm})$; PTOR: perímetro torácico $(\mathrm{cm})$; COM: comprimento do corpo $(\mathrm{cm})$; DOR: comprimento dorso-lombo $(\mathrm{cm})$; GAR: comprimento da garupa (cm); ISQ: distância de ísquios (cm); ILEO: distância de íleos (cm); PE: perímetro escrotal $(\mathrm{cm})$.

W378: Weight standardized at 378 days old ( $\mathrm{kg}$ ); $\mathrm{HH}$ : hip height (cm); CG: chest girth (cm);BL:bodylength (cm); DL:dorsallength (cm); RL: rumplength (cm), DPB: distance of pin bones (cm); DHB: distance of hip bones (cm); SP: scrotal circumference $(\mathrm{cm})$.

Figura 1 - Médias ajustadas de peso (P378), medidas corporais (ATPF, PTOR, COM, DOR, GAR, ISQ, ILEO) e perímetro escrotal (PE) por ano de realização da PGP e rebanho.

Figure 1 - Least squares means for weight (W378), body measurements (WW, HH, CG, BL, DL, RL,DPB, DHB) and scrotal circumference $(S P)$ by year of performance test and herd. 
5,13 e 2,8 para o rebanho NeT, respectivamente.

A característica P378 (Figura 1 e Tabela 2) no rebanho $\mathrm{NeC}$ apresentou coeficiente de regressão linear positivo, porém não-significativo, mostrando haver certa estabilidade de desempenho no decorrer dos anos. A pequena tendência positiva talvez possa ser explicada pelos efeitos ambientais ou pela pequena seleção direcional, conforme constatado por RAZOOK et al. (1993). No rebanho NeS, foi observado coeficiente de regressão linear significativo e mudança genética de cerca de 4,92 kg/ano, o mesmo ocorrendo com o rebanho NeT, com mudança genética de cerca de 3,08 kg/ano. Observa-se que, para os rebanhos selecionados, há tendência sempre crescente para a característica P378. Na Tabela 3, pode-se observar estimativas de ganho genético de 2,71 e 3,08 kg/ano, para os rebanhos NeS e NeT respectivamente.

A característica ATPF, no rebanho $\mathrm{NeC}$, apresentou coeficiente de regressão linear significativo e negativo, contrariamente do que ocorreu com os rebanhos $\mathrm{NeS}$ e NeT, que apresentaram coeficientes positivos, sendo suas respectivas linhas de tendências coincidentes. Pela observação da Tabela 2, pode-se constatar que a característica ATPF obteve significativo aumento genético anual de $0,47 \mathrm{~cm} /$ ano para o rebanho $\mathrm{NeS}$ e $0,52 \mathrm{~cm} /$ ano para o rebanho $\mathrm{NeT}$. Estes valores apresentaram-se, no entanto, superestimados, considerando-se que houve tendência linear negativa com valor de $-0,27 \mathrm{~cm} / \mathrm{ano}$, para a linha controle, bem como coeficientes quadráticos significativos e negativos no rebanho NeT. Na Tabela 3, verificou-se que as estimativas foram de 0,30 a $0,31 \mathrm{~cm} / \mathrm{ano}$, respectivamente para $\mathrm{NeS}$ e $\mathrm{NeT}$, os quais foram menores que os anteriormente citados.

Por meio da análise da Figura 1 e Tabela 2, pode-se observar que a característica perímetro torácico (PTOR), no rebanho $\mathrm{NeC}$, apresentou coeficiente linear negativo e significativo. Para o rebanho NeS, houve efeito quadrático, sendo que a tendência genética foi positiva, de $0,89 \mathrm{~cm} /$ ano. Para o rebanho $\mathrm{NeT}$ observou-se coeficiente quadrático, com valor estimado de tendência genética de $0,59 \mathrm{~cm} /$ ano. Estes valores, embora superestimados, têm certa analogia com os valores médios obtidos para os diferenciais de seleção (CYRILLO et al. 2000), nos quais foram observados valores negativos para $\mathrm{NeC}$, a exemplo de ATPF, e valores maiores para $\mathrm{NeS}$, em relação ao NeT. As estimativas calculadas em função dos desvios (Tabela 3 ) mostraram valores menores da ordem de 0,42 a 0,49, respectivamente, para NeS e NeT.

As características COM, DOR e GAR apresentaram coeficientes lineares negativos e significativos nos três rebanhos estudados, com exceção de DOR no rebanho Nelore Tradicional.

Apesar de COM, DOR e GAR, aparentemente, representarem tendência negativa, pode-se observar que houve ganho genético substancial de 0,$78 ; 0,07$; e $0,32 \mathrm{~cm} /$ ano, para o rebanho $\mathrm{NeS}$, e de 0,$73 ; 0,83$; e $0,19 \mathrm{~cm} /$ ano para o rebanho $\mathrm{NeT}$, respectivamente. As estimativas da Tabela 3 mostram valores correspondentes para COM, DOR e GAR de 0,39; 0,20; e $0,12 \mathrm{~cm} /$ ano do rebanho NeS. Para NeT, estes valores foram 0,$44 ; 0,14 ; \mathrm{e} 0,16 \mathrm{~cm} / \mathrm{ano}$. A exemplo das estimativas pelas equações de regressão, essas também são superestimadas, já que, conforme relatado por CYRILLO et al. (2000), para o comprimento do

Tabela 3 - Tendência genética anual para peso, medidas corporais e perímetro escrotal, considerando-se os desvios médios em relação ao rebanho controle

Table 3 - Annual genetic trends for body weight, body measurements and scrotal circumference, considering average deviation from control herd

\begin{tabular}{lccccccccc}
\hline & \multicolumn{1}{c}{ Característica $^{1}$} \\
Reb $^{2}$ & P378 & ATPF & PTOR & COM & DOR & GAR & ISQ & ILEO & PE \\
Herd $^{2}$ & $W 378$ & $H H$ & $C G$ & $B L$ & $D L$ & $R L$ & $D P B$ & $\begin{array}{c}\text { DHB } \\
(\mathrm{cm})\end{array}$ & $\begin{array}{c}S P \\
(\mathrm{~cm})\end{array}$ \\
& $(\mathrm{kg})$ & $(\mathrm{cm})$ & $(\mathrm{cm})$ & $(\mathrm{cm})$ & $(\mathrm{cm})$ & $(\mathrm{cm})$ & $(\mathrm{cm})$ & 0,12 & 0,09 \\
$\mathrm{NeS}$ & 2,71 & 0,30 & 0,42 & 0,39 & 0,20 & 0,12 & 0,07 & 0,18 & 0,15 \\
$\mathrm{NeT}$ & 3,08 & 0,31 & 0,49 & 0,44 & 0,14 & 0,16 & 0,09 & 0,18 \\
\hline
\end{tabular}

P378: peso padronizado aos 378 dias de idade (kg); ATPF: altura na garupa (cm); PTOR: perímetro torácico (cm); COM: comprimento do corpo (cm); DOR: comprimento dorso-lombo (cm); GAR: comprimento da garupa (cm); ISQ: distância de ísquios (cm); ILEO: distância de íleos (cm); PE: perímetro escrotal $(\mathrm{cm})$.

2 NeC: Rebanho Nelore Controle, NeS: Rebanho Nelore Seleção, NeT: Rebanho Nelore Tradicional.

1 W378: Weight standardized at 378 days old (kg); HH: hip height (cm); CG: chest girth (cm); BL: body length (cm); DL: dorsal length (cm); RL: rump length (cm), $D P B$ : distance of pin bones (cm); DHB: distance of hip bones (cm); SP: scrotal circumference (cm).

2 NeC: Control Herd, NeS: Selection Herd, NeT: Traditional Herd. 
dorso, os diferenciais de seleção secundários foram negativos na população $\mathrm{NeC}$.

Essa tendência, aparentemente negativa para COM e GAR, pode ser, em parte, decorrente dos altos valores das medidas no ano de 1991, mostrados na Figura 1. Estes valores, provavelmente, são decorrentes de erros nas medidas atribuídos a medidores ou mesmo do movimento dos animais mais agitados. O mesmo ocorreu com a característica DOR, com pico observado no ano de 1992.

A característica distância de ísquios (ISQ) apresentou coeficientes de regressão linear e quadrático significativos e positivos para os três rebanhos, com tendência genética de 0,12 e $0,11 \mathrm{~cm} /$ ano para $\mathrm{NeS}$ e NeT, respectivamente. Na Tabela 3, em função dos desvios em relação ao rebanho $\mathrm{NeC}$, os valores de mudança genética anual foram de 0,07 e $0,09 \mathrm{~cm} /$ ano para $\mathrm{NeS}$ e NeT, respectivamente.

A característica distancia de íleos (ILEO) apresentou coeficiente de regressão linear negativo, porém não-significativo, para $\mathrm{NeC}$. Nos rebanhos $\mathrm{NeS}$ e NeT, os coeficientes lineares foram positivos e significativos somente para NeS. Os coeficientes quadráticos foram todos significativos e positivos nos rebanhos selecionados, com tendência genética anual de $0,24 \mathrm{~cm} /$ ano para o rebanho $\mathrm{NeS}$ e $0,12 \mathrm{~cm} / \mathrm{ano}$ para o NeT. Os valores calculados pelos desvios encontrados na Tabela 3 foram de 0,12 e $0,18 \mathrm{~cm} /$ ano para o NeS e NeT, respectivamente.

$\mathrm{Na}$ Figura 1, pode-se visualizar o comportamento anual de perímetro escrotal, nos rebanhos estudados. $\mathrm{O}$ rebanho $\mathrm{NeC}$ apresentou coeficiente de regressão linear negativo e significativo para PE. Já o rebanho $\mathrm{NeS}$ apresentou componente quadrático negativo e significativo, mostrando, porém, certa estabilidade em suas médias. Por outro lado, o rebanho NeT apresentou coeficientes de regressão linear e quadrático negativos e significativos, mostrando declínio nas médias ajustadas com o decorrer dos anos. Em função dessas regressões, as estimativas de tendência genética foram de 0,31 e - $0,21 \mathrm{~cm} / \mathrm{ano}$, respectivamente, em $\mathrm{NeS}$ e $\mathrm{NeT}$.

Observando-se a Figura 1, verifica-se que maiores valores de PE foram encontrados nos anos de 1993 e 1994 de PGP. Isso pode ser atribuído a determinada linhagem de touro de alto valor fenotípico para PE (linhagem Genético), cujas progênies foram avaliadas naqueles anos. Em função da metodologia de reposição de reprodutores do projeto (não mais que dois filhos domesmopai), permaneceram poucos tourinhos dessa linhagem. Conforme verificado em CYRILLO et al. (2000), os diferenciais secundários não diferiram muito da população controle. Levando-se em conta os anos de progênies, essa linhagem de touro (linhagem Genético) foi a grande responsável pela grande porcentagem do desvio, conforme apresentado por CYRILLO et al. (2000). Outra razão do decréscimo pode ter sido a reutilização de touros fundadores, do rebanho NeS, na estação de monta 93/94, reduzindo-se os valores de PE na PGP de 1995 , nesse rebanho.

Pelos valores das herdabilidades e correlações genéticas encontrados, verifica-se que as mudanças genéticas indiretas obtidas, levando-se em conta o rebanho controle, foram compatíveis com a variação genética aditiva das populações estudadas, razão pela qual houve significativa resposta correlacionada nas medidas corporais e perímetro escrotal dos rebanhos selecionados para peso pós desmame.

Os resultados de mudanças genéticas anuais obtidos por meio das análises de regressão têm interpretações e conclusões limitadas, uma vez que para total confiabilidade é necessário primeiro que não ocorra seleção direcional na população controle e, depois, que a tendência fenotípica se apresente principalmente linear, o que não ocorreu, tornando, dessa forma, a maioria das estimativas viesadas e demandando maior acúmulo de informações com o decorrer dos anos.

\section{Conclusões}

As correlações genéticas entre peso padronizado, medidas corporais e perímetro escrotal foram, na maioria, medianas e altas, indicando que genes que determinam a manifestação do peso de animais Nelore são também, em parte, responsáveis pela manifestação de medidas corporais e perímetro escrotal.

As estimativas de herdabilidade para peso padronizado, medidas corporais e perímetro escrotal sugerem que essas características apresentam considerável variação genética aditiva, sendo, portanto, passíveis de seleção direta

A magnitude desses parâmetros na população Nelore de Sertãozinho explica não só a mudança ocorrida no peso, como também as mudanças correlacionadas positivas encontradas nas medidas de várias regiões do corpo dos animais, em função da seleção direta para peso pós-desmame. 


\section{Referências Bibliográficas}

ALENCAR, M.M. de, BARBOSA, P.F., BARBOSA, R.T. et al. 1993. Parâmetros genéticos para peso e circunferência escrotal em touros da raça Canchim. R. Soc. Bras. Zootec., 22(4):572-583.

BOLDMAN, K.G., KRIESE, L.A, VAN VLECK, L.D. et al. 1993. A manual for use for MTDFREML. A set of programs to obtain estimates of variance and covariances [DRAFT]. Lincoln: Departament of Agriculture / Agricultural Research Service. $120 \mathrm{p}$.

BERGMANN, J.A.G., ZAMBORLINI, L.C., PROCÓPIO, C.S.O. et al. 1996. Estimativas de parâmetros genéticos do perímetro escrotal e do peso corporal em animais da raça Nelore. Arq. Bras. Med. Vet. Zootec., 48(1): 69-78.

BROWN, J.E., BROWN, C.J., BUTTS, W.T. 1973. Evaluating relationships among immature measures of size, shape and performance of, beef bulls. II. The relationships between immature measures of size, shape and feedlot traits in young beef bulls. J. Anim. Sci., 37(1):1021.

CYRILLO, J.N.S., FIGUEIREDO, L.A., RAZOOK, A.G. et al. Prova de Ganho de Peso de Sertãozinho: Efeitos genéticos e de ambiente sobre características de crescimento. In: REUNIÃO ANUAL DA SOCIEDADE BRASILEIRA DE ZOOTECNIA, 33, 1996, Fortaleza. Anais... Fortaleza: SBZ, 1996. p.121.

CYRILLO, J.N.S.G. Efeitos da seleção para peso pós-desmame sobre medidas corporais e perímetro escrotal de machos Nelore da Estação Experimental de Zootecnia de Sertãozinho. Jaboticabal, SP: FCAVJ, 1998. 111p. Dissertação (Mestrado em Zootecnia) Faculdade de Ciências Agrárias e Veterinárias de Jaboticabal - UNESP, 1998.

CYRILLO, J.N.S., RAZOOK, A.G., FIGUEIREDO, L.A. et al. 2000. Efeitos da seleção para peso pós-desmame sobre medidas corporais e perímetro escrotal de machos Nelore de Sertãozinho (SP). Rev. bras. zootec., 29(2):444-453.

FERNANDES, A., MAGNABOSCO, D.U., OJALA, M. et al. Estimativas de parâmetros genéticos e ambientais de medidas corporais e peso em bovinos da raça Brahman nos trópicos. In: REUNI $\tilde{O} O$ ANUAL DA SOCIEDADE BRASILEIRA DE ZOOTECNIA, 33, 1996, Fortaleza. Anais...Fortaleza: SBZ, 1996. p.136.

GONÇALVES, J.N.S., FIGUEIREDO, L.A., RAZOOK, A.G. et al. Prova de ganho de peso de Sertãozinho: efeitos genéticos e de ambiente sobre características de crescimento. In: REUNIÃO ANUAL DA SOCIEDADE BRASILEIRA DE ZOOTECNIA, 33, 1996, Fortaleza. Anais... Fortaleza: SBZ, 1996. p.121.

HARVEY, W.R. 1990. User's guide for LSMLMW (Mixed Model Least-Squares and Maxium Likelihood Computer Program). Wooster: Ohio State University. 91p.
KOOTS, K.R., GIBSON, J.P., SMITH, C. et al. 1994. Analyses of published genetic parameters estimates for beef production traits. 1-Heritability. Anim. Breed. Abstr., 62:309-338.

LIMA, F.P., BONILHA NETO, L.M., RAZOOK, A.G. et al. 1989. Parâmetros genéticos em características morfológicas de bovinos Nelore. Bol. Ind. Anim., 46(2): 249-257.

MAGNABOSCO, C.U., OJALA, M., FERNANDES, A. et al. Efeitos de fatores ambientais sobre medidas corporais e peso em bovinos da raça Brahman no México. In: REUNIÃO ANUAL DA SOCIEDADE BRASILEIRA DE ZOOTECNIA, 33, 1996, Fortaleza. Anais... Fortaleza: SBZ, 1996. p.139.

MERCADANTE, M.E.Z. Estimativas de parâmetros genéticos para características de crescimento de zebuínos de corte. uma revisão. Ribeirão Preto, SP: FMRP, 1994, 66p. Exame de Qualificação. Faculdade de Medicina de Ribeirão Preto/ Universidade de São Paulo, 1994.

MOHIUDDIN, G. 1993. Estimates of genetic and phenotypic parameters of some performance traits in beef cattle. Anim. Breed. Abstr., 61(8):495-522.

MRODE, R.A. 1988. Selection experiments in beef cattle. Part 2: A review of responses and correlated responses. Anim. Breed. Abstr., 56(3):155-167.

RAZOOK, A.G., FIGUEIREDO, L.A., BONILHA NETO, L.M. et al. 1993. Intensidade de seleção e respostas direta e correlacionadas em 10 anos de progênies de bovinos das raças Nelore e Guzerá para peso pós-desmame. Bol. Ind. Anim., 50(2):147-163.

RAZOOK, A.G., FIGUEIREDO, L.A., CYRILLO, J.N.S.G. et al. 1997. Prova de ganho de peso. Normas adotadas pela Estação Experimental de Zootecnia de Sertãozinho. Nova Odessa, Instituto de Zootecnia. 33p. (Boletim Técnico n ${ }^{\circ} 40$ ).

WINKLER, R. Tamanho corporal e suas relações com algumas características reprodutivas em fêmeas bovinas adultas da raça Guzerá. Belo Horizonte, MG: UFMG, 1993. 116p. Dissertação (Mestrado em Zootecnia) - Escola de Veterinária, 1993.
Recebido em: 17/05/99

Aceito em: 26/06/00 